\title{
summary
}

\section{Hydrophilic primer has double the risk of bracket bond failure}

\begin{abstract}
Littlewood SJ, Mitchell L, Greenwood DC. A randomized controlled trial to investigate brackets bonded with a hydrophilic primer. J Orthod 2001; 28:301-305
\end{abstract}

Question: In patients who need orthodontic bracketing is a hydrophilic primer or a conventional primer more effective for bonding?

Objective To compare clinical failure rates of brackets bonded with a prototype hydrophilic primer and conventional primer.

Design Randomised controlled trial (RCT) in a hospital setting. Intervention A split mouth design was used. Sides were randomly allocated to either hydrophilic (supplied by 3M Unitek (Monrovia, California, USA) or conventional primer (conventional Transbond $^{\mathrm{TM}} 3 \mathrm{M}$ Unitek) adhesive), with the other side having alternative primer. Brackets were applied to 33 patients using a standard procedure. A total of 266 bonds were completed with each primer.

Outcome measures Subjects were followed for 6 months and site of failure, type of primer and time since bonding were recorded.

Results All 33 patients were seen after 6 months The hydrophilic primer had an increased risk of bracket failure (see Table), with a hazard ratio of 2.2 (95\% confidence interval, 1.1-4.5).
Table 1 Bond failure according to primer

\begin{tabular}{lll}
\hline & $\begin{array}{c}\text { Patients with at least one } \\
\text { bond failure }\end{array}$ & Bonds failed \\
\hline Hydrophilic primer & $27(82.0 \%)$ & $50(18.8 \%)$ \\
Conventional primer & $15(45.0 \%)$ & $18(6.8 \%)$ \\
\hline
\end{tabular}

Conclusions Brackets bonded with the prototype hydrophilic primer had double the risk of failure compared with conventional primer over 6 months. Hydrophilic primer cannot be recommended for clinical use.

Evidence-Based Dentistry (2002) 8, 52. doi:10.1038/sj. ebd.6400107

Address for reprints: SJ Littlewood, Department of Orthodontics, St Luke's Hospital, Little Horton Lane, Bradford, Yorkshire, UK.

\section{Commentary}

Readers of orthodontic journals are fortunate to encounter such a pragmatic, clearly structured and easily understood scientific paper. The authors have applied sound methodological criteria to test for a clearly focused hypothesis. Sample size required to show statistically significant differences was calculated pre-hoc. Consent to participate was obtained from each patient entering the trial and follow-up was completed without any withdrawals. Blinding was performed where possible. The observations were thus formed from a RCT accounting for the multiple and inter-individual causes of bond failure such as bite force or orthodontic force application, by the use of a split mouth design.
Previous studies conducted with the conventional primer resulted in similar bond failure rates to the subgroup in this study. Therefore it can be concluded that the operator applied a standard technique that did not bias the outcome.

Moisture-insensitive primers are designed for the purpose inherent in their name. The authors themselves have acknowledged in a previous article that bond strength of conventional materials was reduced by as much as $50 \%$ in the presence of moisture whereas the bond strength of moisture-insensitive primers remained unchanged. ${ }^{1}$ Thus the conclusions of the authors that, "the hydrophilic primer cannot therefore be recommended for clinical use" should be supplemented by the phrase, "in situations where moisture control is attainable".
Altogether this is a paper well worth reading, and should be an inspiration to orthodontic researchers because of its clear methodology. As the hydrophilic primer tested in this study is a prototype, orthodontic practitioners asking for guidelines for everyday practice might be well-advised to look out for the followup research examining the next generation of moisture-insensitive primers.

1. Littlewood SJ, Mitchell L, Greenwood DC, Bubb NL, Wood DJ. Investigation of a hydrophilic primer for orthodontic bonding: an in vitro study. J Orthodont 2000; 27:181-186.

Dieter Brothag Department of Orthodontics, University of Mainz, Mainz, Germany 